ISSN 1996-1944

www.mdpi.com/journal/materials

Review

\title{
Morphologies, Preparations and Applications of Layered Double Hydroxide Micro-/Nanostructures
}

\author{
Ye Kuang ${ }^{1}$, Lina Zhao ${ }^{1}$, Shuai Zhang ${ }^{2}$, Fazhi Zhang ${ }^{1}$, Mingdong Dong ${ }^{2, *}$ and Sailong Xu ${ }^{1, *}$ \\ 1 State Key Laboratory of Chemical Resource Engineering, Beijing University of Chemical \\ Technology, 15 Beisanhuan East Road, Chaoyang District, Beijing, 100029, China \\ 2 Interdisciplinary Nanoscience Center (iNANO) and Department of Physics and Astronomy, Aarhus \\ University, DK-8000 Aarhus C, Denmark
}

* Author to whom correspondence should be addressed; E-Mails: xusl@ mail.buct.edu.cn (S.X.); dong@iano.au.dk (M.D.).

Received: 28 October 2010; in revised form: 29 November 2010 / Accepted: 3 December 2010 / Published: 9 December 2010

\begin{abstract}
Layered double hydroxides (LDHs), also well-known as hydrotalcite-like layered clays, have been widely investigated in the fields of catalysts and catalyst support, anion exchanger, electrical and optical functional materials, flame retardants and nanoadditives. This feature article focuses on the progress in micro-/nanostructured LDHs in terms of morphology, and also on the preparations, applications, and perspectives of the LDHs with different morphologies.
\end{abstract}

Keywords: layered double hydroxides; morphology; preparation; application

\section{Introduction}

Nanostructured materials have generated diverse scientific and technological interest in many potential areas of applications such as energy conversion, electronics, catalysis, optics, chemical sensing, and medicine. As one type of layered material, such as anionic clays (e.g., layered double hydroxides) and cationic clays (e.g., montmorillonite), layered double hydroxides (LDHs) [1-4] are a class of anionic clays with the structure based on brucite $\left(\mathrm{Mg}(\mathrm{OH})_{2}\right)$-like layers in which some of the divalent cations have been replaced by trivalent cations yielding positively charged sheets. LDHs can be represented by the general formula $\left[\mathrm{M}^{\mathrm{II}}{ }_{1-x} \mathrm{M}^{\mathrm{III}}{ }_{x}(\mathrm{OH})_{2}\right]^{x+}\left(\mathrm{A}^{n-}\right)_{x / n} \cdot y \mathrm{H}_{2} \mathrm{O}$, where $\mathrm{M}^{\mathrm{II}}$ and $\mathrm{M}^{\mathrm{III}}$ cations 
occupy octahedral holes in a brucite-like layer and $\mathrm{A}^{n-}$ anion is located in the hydrated interlayer galleries. The identities of the divalent and trivalent cations ( $\mathrm{M}^{\mathrm{II}}$ and $\mathrm{M}^{\mathrm{III}}$, respectively) and the interlayer anion $\left(\mathrm{A}^{n-}\right)$, together with the value of the stoichiometric coefficient $(x)$, may be varied over a wide range, giving rise to a large class of isostructural materials. Considered generally as promising materials [5-9] in view of their high chemical versatility associated with a tunable anionic exchange capacity, LDHs are widely used in commercial products as adsorbents, catalyst support precursors, anion exchangers, acid residue scavengers, flame retardants and polymer stabilizers. LDH powders, prepared by conventional coprecipitation, typically show preferential growth of $a b$ faces (i.e., perpendicular to the stacking direction) and microscale hexagonal platelet morphology [10]. Due to the highly active surface atoms, LDH powders are spontaneously ready to aggregate during storage and application, thus leading to limitations in their technical applicability. Therefore, it is of great interest and importance to generate alternative predefined structures.

Important advances in LDH morphology have been made in the past years, ranging from $\mathrm{LDH}$ powders [11,12], spheres [13-18], nanosized LDH belt [19], fibrous structure [20], to LDH films on substrates [21-27]. In this feature article, we summarize the progress in fabrication of LDH structure materials with different morphologies from powder/sphere, one-dimensional (1-D) belt/fiber; to 2-D films. In addition, we focus on the applications of LDHs and offer some perspectives for future multifunctional LDH materials.

\section{LDH Morphology}

\subsection{Powdery LDHs}

Powdery LDHs typically show the brucite $\left(\mathrm{Mg}(\mathrm{OH})_{2}\right)$-like layered structure via a conventional co-precipitation process. When synthesized by a hydrothermal approach induced by slow hydrolysis of urea, the resulting MgAl-LDH usually exhibits hexagonal platelet morphology at the micro scale (Figure 1); this suggests the preferential growth of $a b$ faces (i.e., perpendicular to the stacking direction). The platelets are often observed to aggregate [10]. The microsized platelets can be fairly well prepared into nanosized crystals. Gursky et al. [12] obtained nanosized colloidal MgAl-LDH particles through coprecipitation from metal salts dissolved in methanol in the presence of $\mathrm{NaOH}$. The approach was extended to prepare $\mathrm{Al}^{3+}$-based LDHs, such as CoAl-, ZnAl-, and NiAl-LDH. $\mathrm{Xu}$ et al. [11] also prepared colloidal MgAl-LDH by a fast coprecipitation followed by controlled hydrothermal treatment (Figure 2). The obtained LDH platelets had the dimensional sizes ranging from 50 to $300 \mathrm{~nm}$. The approach was also extended to prepare other types of LDHs, including transition metal such as $\mathrm{Ni}^{2+}, \mathrm{Fe}^{2+}, \mathrm{Fe}^{3+}$, and $\mathrm{Gd}^{3+}$. To control the growth environment of $\mathrm{LDH}$, such as by using microemulsion, O'Hare et al. [28-31] prepared very small LDH particles in isooctane-sodium dodecyl sulfate/aqueous solution and also obtained LDH monolayer with a thickness of $14 \AA$. In addition, nanosized LDH platelets can be synthesized in a large-amount production using a method involving separate nucleation and aging steps (SNAS) [32-34], the key features of which are a very rapid mixing and nucleation process in a modified colloid mill followed by a separate aging process. The major advantages of this method for the preparation of LDHs can be summarized as follows: (1) the synthesis process is simple to carry out, (2) small crystallite sizes can be obtained, (3) the crystallite size 
distribution of the product is much narrower than that obtained using conventional coprecipitation methods. With regard to types of LDHs, general LDHs are able to be prepared, such as $\mathrm{Al}^{3+}$-based and transmission metal cation-based LDHs. Using this method, numerous nanosized LDH building blocks were prepared, even in an organic/water solvent system for preparation of CaAl-LDH nanoplatelets without byproduct of calcium carbonate, which could be attributed to the ability of organic media (such as ethanol) to prevent carbon dioxides from entering the reaction solution [34].

Figure 1. (a) Schematic illustration of the layered double hydroxide (LDH) structure showing the metal hydroxide octahedra stacked along the crystallographic $c$ axis (indicated as a red arrow). Water (grey) and anions (pink) are present in the interlayer region. The green parts correspond to $\mathrm{M}^{\mathrm{II}}$ cations and the red dots to $\mathrm{M}^{\mathrm{III}}$ cations. (b) Scanning electron microscope (SEM) image of typical LDH crystals.

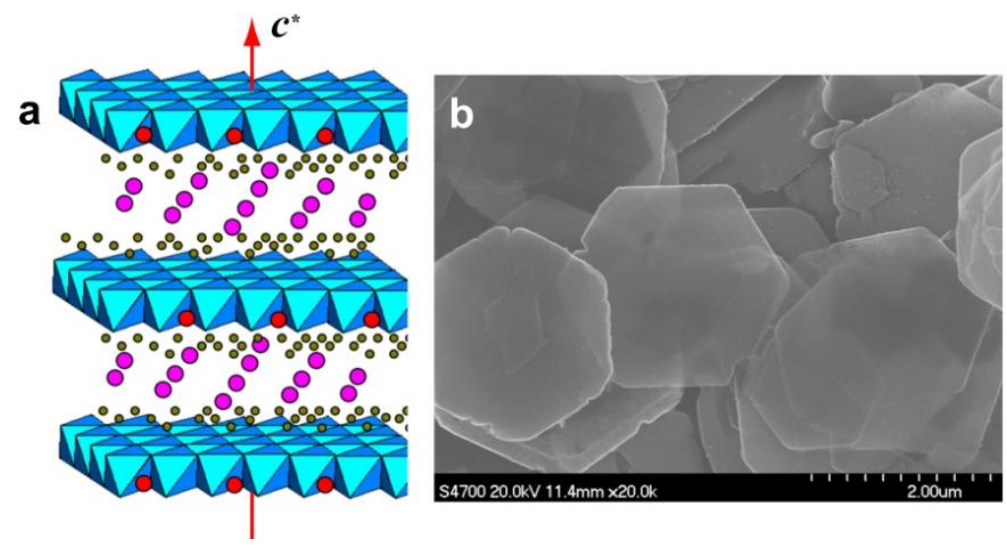

Figure 2. Dispersion of an LDH aggregate (A) into several smaller LDH aggregates (B) via an aging process, or $(\mathbf{C})$ both $(\mathrm{A}$ and $\mathrm{B})$ into individual LDH nanosheet crystallites via the new hydrothermal treatment method. Reprinted with permission from [11], (C) American Chemical Society, 2005.

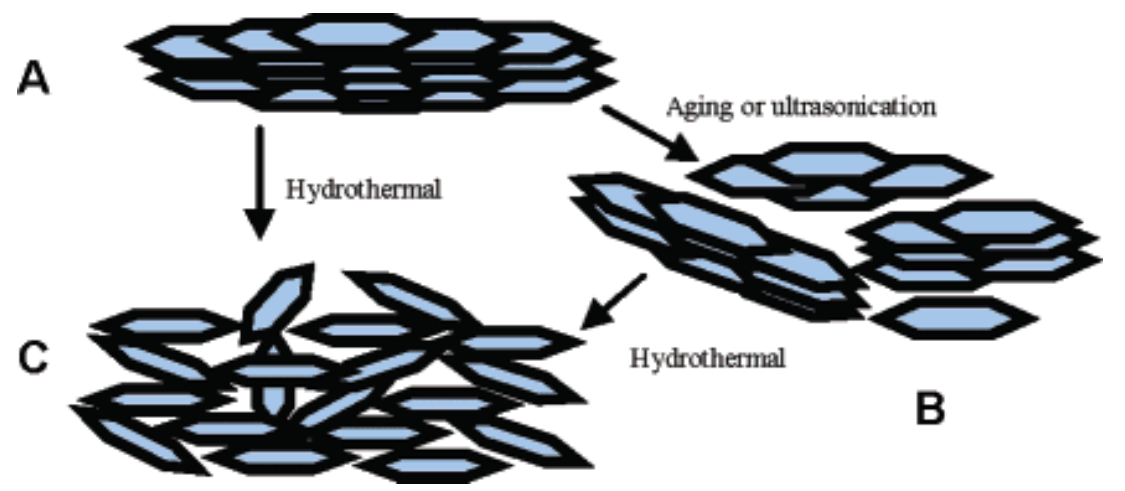

\subsection{Spherical LDHs}

Spherical structure is of interest for a number of different applications, such as catalysts, sorbents, lithium-ion battery electrodes, and carriers for cellular drug and gene delivery. One main synthesis route-template-directed synthesis-has been performed to prepare spherical LDH structure, involving either soft templates or hard templates. The soft templates, for example spherical vesicle 
formed by sodium dodecanesulfonate (SDS), were used to synthesize micrometer-sized rosette-like solid LDH spherical aggregates with SDS anions intercalated already in the LDH galleries, by urea hydrolysis under high-temperature hydrothermal treatment of $150{ }^{\circ} \mathrm{C}$ [16]. Coral-like MgAl-LDH solid microsphere was also synthesized using ethylene glycol/methanol/dodecyl sulfate nonaqueous polar solvent/surfactant system [13]. The hard template method, using hard cores such as polystyrene (PS) beads as templates, involves formation of the LDH hollow sphere with nanoshell face-on orientation. In such a method, positively charged colloidal LDH nanoplatelets were necessarily prepared, and then deposited or assembled as building block on the surface of PS latex [17], carbon [15], and silica [18] (Figure 3), giving the LDH shell walls. The necessity of preparing positively charged LDH was achieved either by synthesizing LDH nanoparticles using a protocol involving a fast coprecipitation followed by controlled hydrothermal treatment [11], or by obtaining LDH nanosheets via delamination of $\mathrm{Al}^{3+}$-based types of LDHs (such as CoAl-, ZnAl-LDH, and MgAl-LDH) in formamide [35-38]. Compared with the soft template approach, the hard template could have advantages in facile preparation of size-controllable hollow spheres due to the rich functional groups on the surface.

Furthermore, note that with the aid of the template of 3-D close-packed PS array, a three-dimension open macroporous (3DOM) LDH framework was prepared during a process of coprecipitation $[39,40]$.

Figure 3. Synthesis of the magnetic $\mathrm{Fe}_{3} \mathrm{O}_{4}$ core/LDH shell composite structure. Reprinted with permission from [18], (C) Wiley-VCH, 2009.

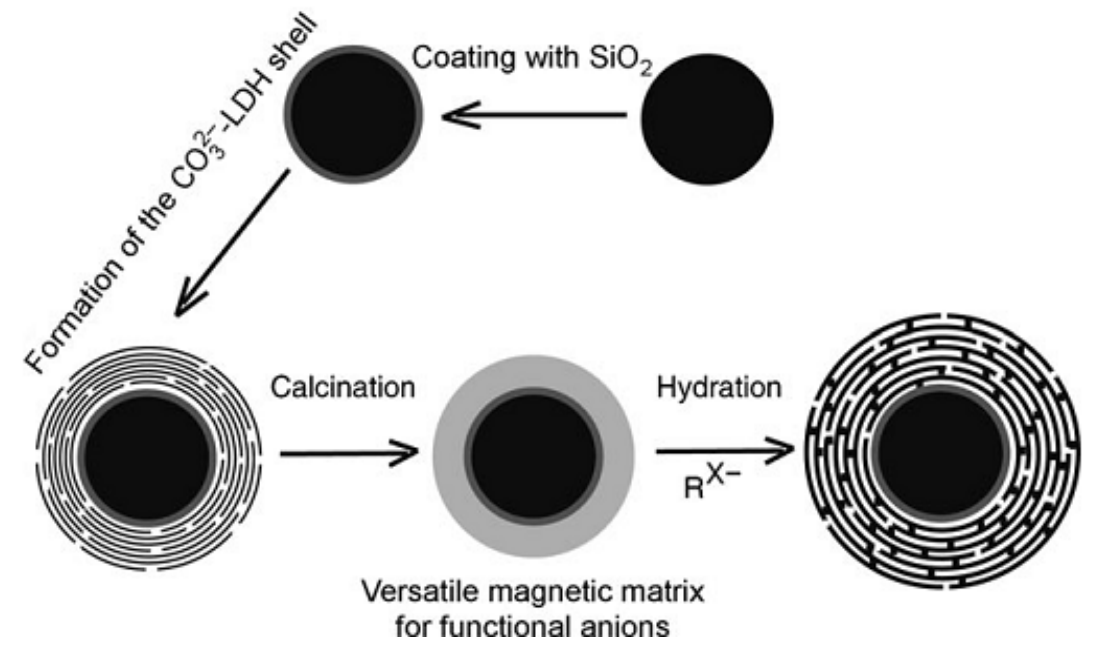

\subsection{1-D LDHs Nanostructures}

LDHs are well-known to show preferential growth perpendicular to the stacking direction, and therefore it is still a challenge to engineer microsized LDH platelet into 1-D structures. This type of rigid platelet is not similar to a graphene sheet which is able to be rolled into a tube. In few previous studies [41-43], 1-D LDH structures were obtained through the control of nucleation and growth of LDH crystal, such as nanobelt and fibrous structure. Zhang et al. synthesized MgAl-LDHs with a high aspect ratio via calcination and rehydrolysis under hydrothermal conditions [41]. Zhao et al. prepared MgAl-LDH nanowires and nanorods via hydrothermal treatment at a high temperature and $\mathrm{ZnCoFe}-\mathrm{LDH}$ nanowires with controllable morphology in a water-in-oil microemulsion [42,43]. Interestingly, O'Hare' group obtained MgAl-LDH belt structure in a water-in-oil reverse 
microemulsion system (Figure 4) [19]. The reverse system involved was considered to offer two important water pools in the confined nucleation and growth: small spheroidal microemulsion spontaneously formed by SDS act as microreactors for the nucleation and crystallization of LDHs, and large branching reservoirs contributing to confine LDH growth that is distinctly different from the traditional coprecipitation with a preferred orientation along the $c$ axis [19]. Recently, Lima et al. fabricated fibular LDH via folding of LDH layers by the aid of SDS surfactant [20]. The possible mechanism is that mixed oxides $((\mathrm{Mg}, \mathrm{Al})-\mathrm{O})$ recover the layered structure typical of hydrotalcites in the presence of SDS solutions, and then the most external layers in a particle of LDH fold to give rise to a mesostructured material by the formation of small amounts of $\mathrm{Al}-\mathrm{OH}-\mathrm{Al}$ groups (Figure 5). The local disturbance in the layered structure was thereby proposed as an evolution from platelet-like to fibrous pipe. Comparison of all the 1-D LDH structures in the above studies shows the dependence of the formation on relatively restricted conditions necessary to form vesicles, micelles, or reverse emulsions, since the weak liquid confinements are easily deformed and coalesced during synthesis.

Figure 4. (A) SEM image of a belt-like LDH structure with the parallel linear pattern highlighted in the white rectangle. (B) TEM image of one belt reveals the lamellar structure; a HRTEM image of this structure is shown in the inset, and (C) spectrum of the chemical compositions analyzed by energy dispersive X-ray (EDX). The belts were easily exfoliated to give structures shown by the SEM image in (D) and TEM image in (E). (F) The corresponding selective area electron diffraction pattern. Reprinted with permission from [19], @ American Chemical Society, 2005.
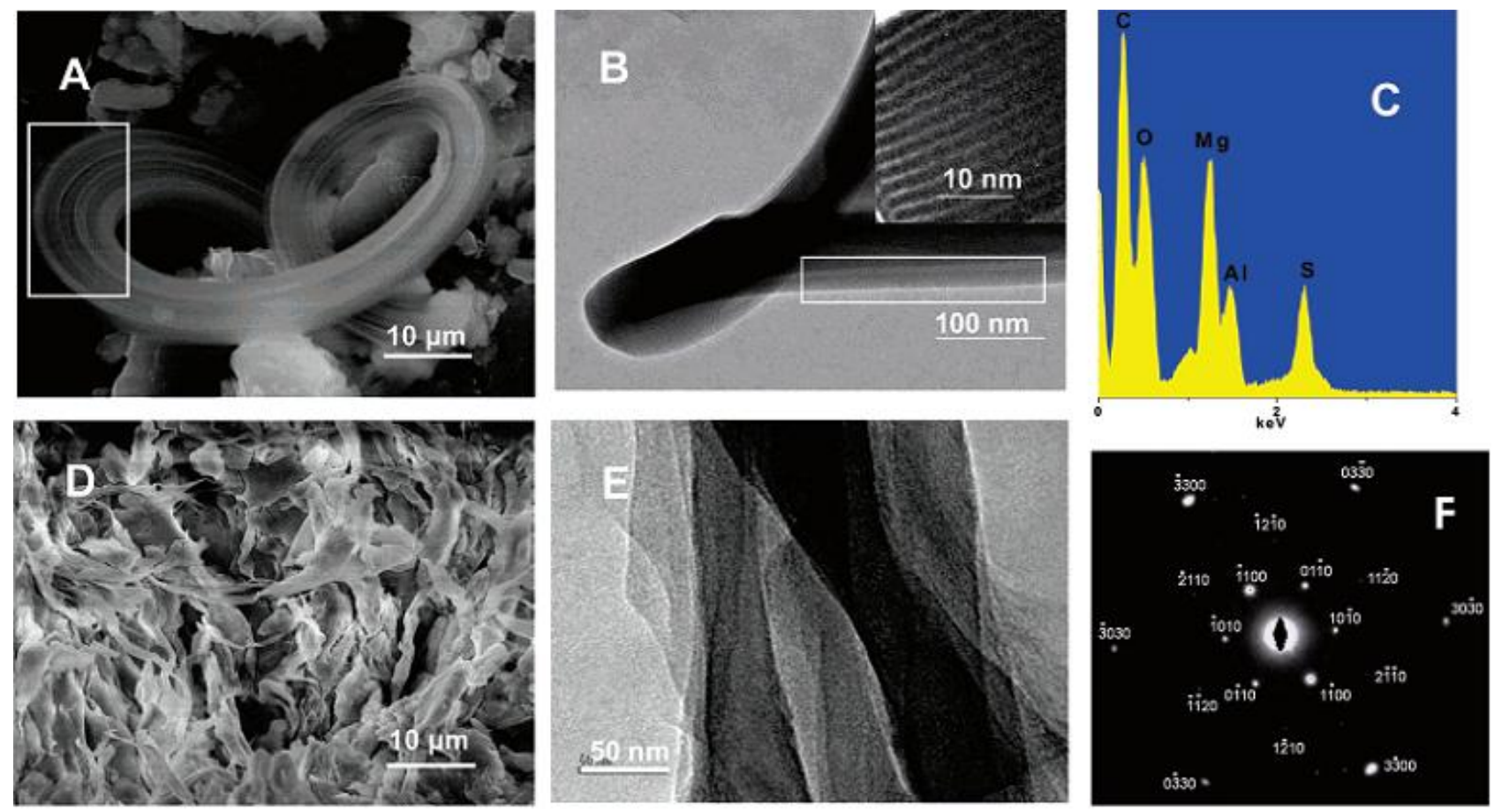
Figure 5. Possible mechanism for the folding of layers of LDH to give fibrous hydrotalcites. Reprinted with permission from [20], (C) American Chemical Society, 2008.

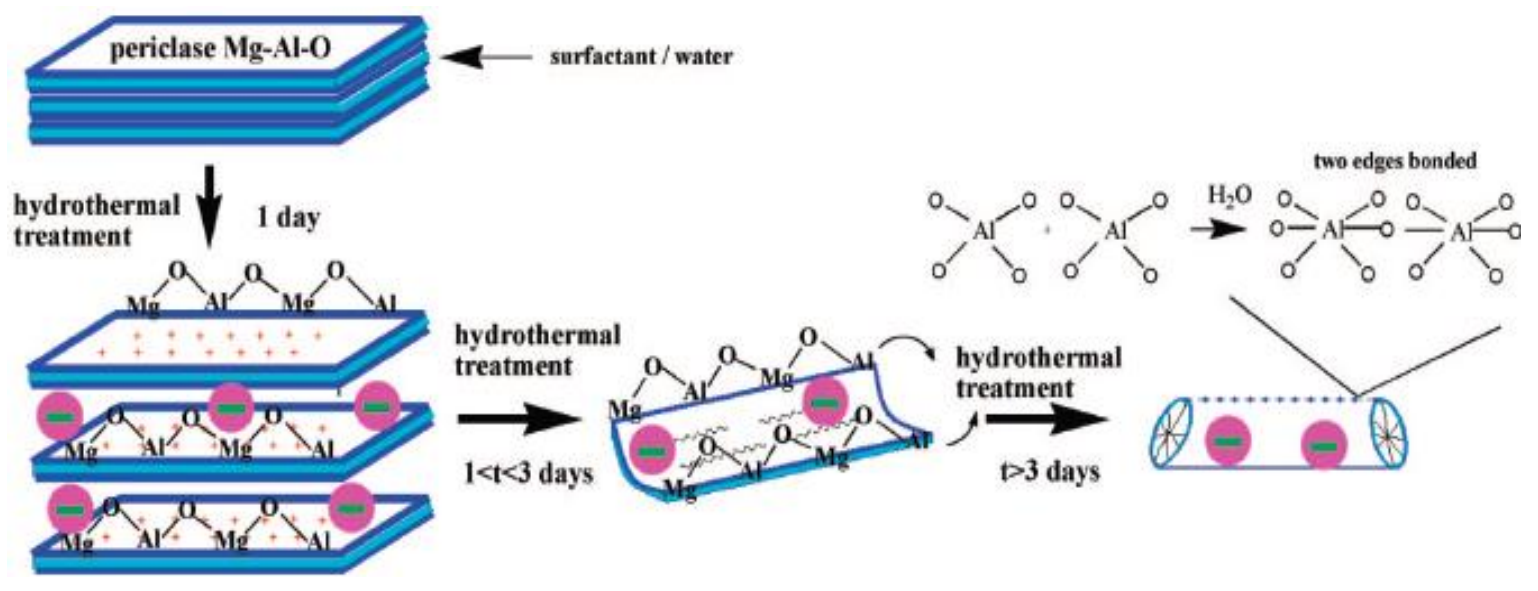

\subsection{2-D LDH Films}

LDH films have increasingly attracted attention due to advantages in the context of immobilization of LDH structures. One advantage of fabrication of well-oriented or self-supporting LDH films is to avoid spontaneous aggregation of LDH powders. Previous studies have shown the fabrications of LDH films with microsized LDH platelets oriented perpendicularly or horizontally to the underlying metal or surface-modified substrates [21-26,44-62]. With regards to orientation, 2-D LDH films can be classified into two main types: perpendicular and parallel oriented.

\subsubsection{Parallel-Oriented LDH Films}

Parallel-oriented LDH films usually consist of face-on oriented building blocks of platelet-like LDHs. The face-on orientation of the building blocks of LDH platelets are achieved through various methods, such as solvent evaporation [44-50], electrochemical deposition [51-53], spin-coating technique [54], Langmuir-Blodgett (LB) technique [55], Layer-by-layer (LbL) technique [35-38], and in-situ growth [27].

Solvent evaporation and colloid deposition technique are the direct ways to prepare LDH films. LDH platelets or exfoliated LDH nanosheets are evenly dispersed in solvent (such as methanol, ethanol, and water) and assembled with the solvent evaporation. Iyi et al. prepared water-swellable MgAl-LDH film by solvent evaporation on polyethylene (PE) substrate and obtained self-supported film of thickness in the range from 10 to $25 \mu \mathrm{m}$ [44]. Wang et al. fabricated transparent ZnAl- and NiAl-LDH films by solvent evaporation of LDH powder obtained by the SNAS method [45]. Using colloid deposition, Itaya et al. prepared film of $100 \mathrm{~nm}$ thickness on a $\mathrm{SnO}_{2}$ electrode [46]. Similarly, Lee et al. prepared the monolayer [47,48], the multilayer [49] (Figure 6), and transparent hybrid layer [50] on substrates - all were assembled from LDH nanoplatelets. Electrochemical deposition technique is also used to prepare LDH films due to the positive charge of LDH colloid. By the aid of this method, ZnAl-LDH, CoAl-LDH, and NiAl-LDH films were prepared on different electrode surfaces [51-53]. Spin coating is another effective approach for fabrication of inorganic films with controlled structure and crystal orientation. Zhang et al. reported that MgAl-LDH films was readily 
prepared by spin coating nanodispersed MgAl-LDH solution on a magnesium-containing alloy substrate [54]. In addition, LB technique was utilized to fabricate MgAl-LDH film using an anionic $\mathrm{Ru}(\mathrm{II})$ cyanide polypyridyl complex as template [55].

Figure 6. SEM images of the monolayer of LbL LDH nanocrystals: 1 layer (L) (a); 3 L (b); and $5 \mathrm{~L} \mathrm{MgAl-LDH/Si} \mathrm{(c);} \mathrm{Cross-sectional} \mathrm{views} \mathrm{of} 1 \mathrm{~L}(\mathbf{d}) ; 2 \mathrm{~L}(\mathbf{e}) ; 3 \mathrm{~L} \mathrm{(f)} ; 4 \mathrm{~L}$ (g) and $5 \mathrm{~L}$ (h) samples. Reprinted with permission from [49], () American Chemical Society, 2007.

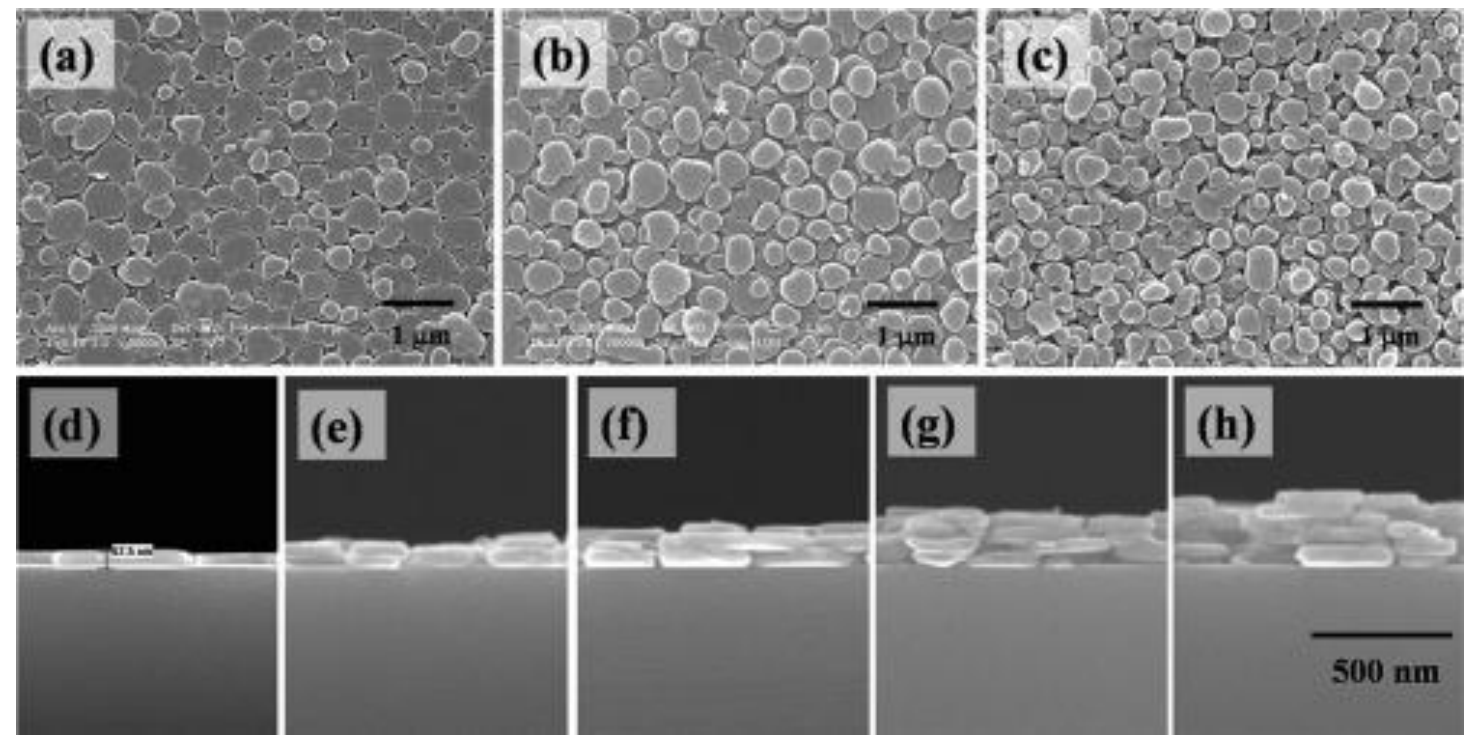

Figure 7. Schematic illustration of the possible delamination mechanism for LDHs in formamide, which were used as positively charged building block to prepare multilayer film of (LDH/PSS)n assembled on a quartz glass substrate. Reprinted with permission from [58], (C) American Chemical Society, 2006.

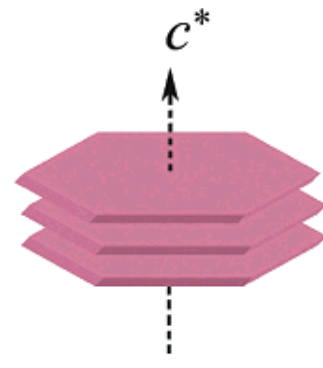

LDH crystal
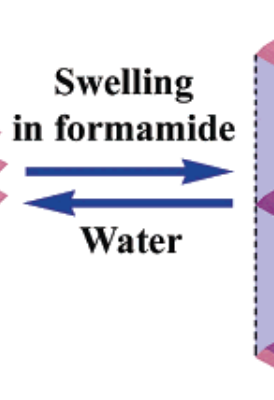

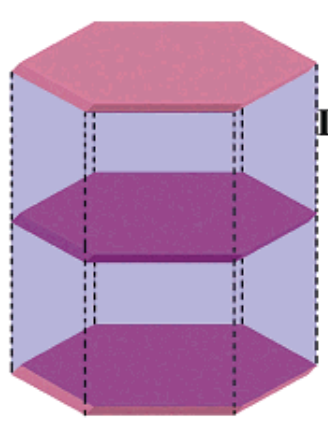

swollen phase

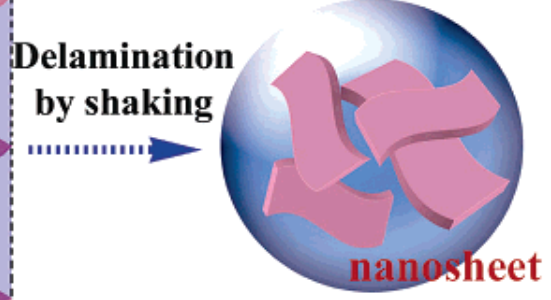

colloidal suspension

Compared with the above methods, LbL assembly is a commonly used approach to prepare LDH films. This approach is based on assembly of alternating deposition of oppositely charged building blocks either mainly via electrostatic interaction [35], to which Sasaki and co-workers have made a pioneer contribution, or via hydrogen bonding [38]. Through assembly of positively charged LDH building blocks and negatively charged polyelectrolytes, a serials of LDH nanosheet/polyelectrolyte heterogeneous ultrathin films were successfully prepared [35-37], such as CoAl-LDH/poly(sodium 4styrenesulfonate) [35,37] (Figure 7) and MgAl-LDH/poly(p-phenylene) anionic derivate ultrathin films 
[36]. Typically, the LDH nanosheets utilized were obtained via exploitation of $\mathrm{Al}^{3+}$-based type (such as CoAl- and MgAl-LDH) in formamide, as mentioned in Section 2.2.

In situ growth is also an effective method to fabricate parallel-oriented LDH films whose crystal is directly formed on the surface of the substrate. One feature of the approach lies in the strong adhesion between the LDHs and the substrates. We have recently shown a parallel-oriented LDH structure with LDH $c$-axis perpendicular to the glass substrate upon surface modification of poly(vinyl alcohol) (PVA) [27]. The X-Ray diffraction (XRD) pattern of the LDH film showed the absence of any non-basal reflections $(h, k \neq 0)$ at high angle, instead of basal $(00 l)$ reflections with a series of LDH peaks appearing as narrow, symmetric, strong lines at low angles. The hydrogen bonding between PVA and the hydroxyl groups of LDH crystallites orientation was proposed for the formation of the orientation.

\subsubsection{Perpendicular-Oriented LDH Films}

Perpendicular-oriented LDH films are usually composed of edge-on oriented LDH platelets. Such films were typically prepared using in situ growth based on a hydrothermal synthesis, which is mentioned in Section 2.4.1 to fabricate parallel oriented LDH films. Lei et al. reported the edge-on oriented MgAl-LDH thin films with microscale thickness on the sulfonated PS flat substrates by urea hydrolysis reaction at $70-80{ }^{\circ} \mathrm{C}$ over more than eight days [56]. Further investigation of growth kinetics of LDH microcrystals supported the possibility of templating the formation of the edge-on orientated MgAl-LDH film [57]. Using the approach, Gao et al. reported the preparation of a ZnAl-LDH film on single Al substrate [22]. As an alternative, Chen et al. presented the preparation of NiAl-LDH films by using single porous anodic alumina/aluminum (PAO/Al) foil as both substrate and sole source of aluminum [21]. This opened up the possibility of growing large-scale uniform LDH films directly from substrates. Zhang et al. further demonstrated the possibilities of micro/nanostructured ZnAl-LDH film precursors, with (Figure 8) or without the necessity of the pre-treatment of anodization to $\mathrm{Al}$ substrate [25,26]. More interestingly, using two substrates (Al and $\mathrm{Zn}$ or $\mathrm{Cu}$ foils) as trivalent and bivalent resources, respectively, Huang's group fabricated $\mathrm{ZnAl}$ - and CuAl-LDH films on the Al substrates [24]. Recently, Zhao et al. reported the fabrication of hierarchical ZnAl-, NiAl-, and MgAl-LDH films on intriguing man-made supports (article, cloth and sponge) through a combined procedure of sol-gel process and in situ growth method [58]. All the involved LDH structures observed by scanning electron microscope (SEM) in the above studies showed that the building blocks, typically microsized LDH platelets, were observed perpendicular to the underlying surfaces of the substrates. The XRD patterns involved, however, show the different results of the presence or absence of basal and higher order $(00 l)$ reflections at low angle. This could be attributed to the difference in orientations between vertically oriented and titled LDH platelets, which could deserve further research in the field of inorganic materials. 
Figure 8. SEM of the ZnAl-LDH-laurate hybrid film showing the hemispherical protrusions. ZnAl-LDH-La/Al. The inset shows the image of contact angle of a water droplet on the surface of the ZnAl-LDH-laurate hybrid film.

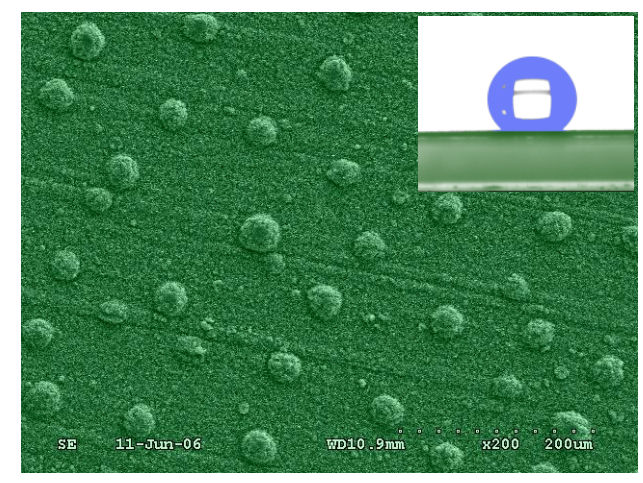

On the basis of the above-mentioned LDH micro-/nanostructures, an overview of the morphologies in terms of dimension is given in Table 1 .

Table 1. Overview of the LDH micro-/nanostructures with different dimensions and synthesis.

\begin{tabular}{|c|c|c|c|}
\hline Dimension & Morphology & Method & References \\
\hline \multirow{2}{*}{$0-\mathrm{D}$} & powder & SNAS, coprecipitation, hydrothermal & $28-34$ \\
\hline & sphere & LbL, coprecipitation & $15-18$ \\
\hline \multirow{4}{*}{$1-\mathrm{D}$} & high aspect ratio & hydrothermal & 41 \\
\hline & nanowire & calcination and rehydrolysis, hydrothermal & 42,43 \\
\hline & nanobelt & coprecipitation & 19 \\
\hline & nanotube & coprecipitation & 20 \\
\hline \multirow{2}{*}{ 2-D } & parallel-oriented & $\begin{array}{l}\text { solvent evaporation, electrochemical deposition } \\
\text { spin-coating, LB, LbL, in-situ growth }\end{array}$ & $44-59$ \\
\hline & $\begin{array}{c}\text { perpendicular- } \\
\text { oriented }\end{array}$ & in-situ growth & $21-26,60-62$ \\
\hline 3-D & $3 \mathrm{DOM}$ & coprecipitation & $39-40$ \\
\hline
\end{tabular}

\section{Application of LDHs}

The nanostructures involved often combine fascinating shapes with remarkable properties. With the introduction of new techniques and novel concepts for preparation of LDH structures, together with the requirements of practical applications, various studies focus on the properties and applications of nanostructured LDHs.

\subsection{Catalysts}

LDH powder, LDH film, and activated rehydrated LDH (RLDH) structures are well-known as effective solid catalysts. Zhang et al. used the $\mathrm{Cu}^{2+}$-based $\mathrm{LDH}$ powder as a catalyst for wet oxidation [59], and reported that the array of active $\mathrm{Cu}^{2+}$ centers on the surface of CuZnAl-CLDH is ordered net shaped, which is influenced by the effect of ordered cross trap. The advantages of novel LDH films are able to overcome the problems of use of powdery catalysts on an industrial scale, such 
as high pressure drop and difficult catalyst separation. Lv et al. reported that the activated MgAl-LDH film was promising to be used as precursor to synthesize monolithic catalyst for the aldol condensation of acetone and other base catalyzed reactions [60]. For the interesting 3DOM LDH structure, Géraud et al. demonstrated that the activated $\mathrm{MgAl}-\mathrm{LDH}$, with decatungstate anion intercalated exhibited the enhanced photocatalytic activity for the photodegradation of 2, 6-dimethylphenol with control of the corresponding coprecipitated LDH material [40], due to the high specific surface of the novel 3DOM structure.

\subsection{Water Treatment}

Waste water often contains oxyanions such as $\mathrm{F}^{-}, \mathrm{Cl}^{-}, \mathrm{Br}^{-}, \mathrm{PO}_{4}{ }^{3-}$, which are harmful to both humans and wildlife. Enhanced ability to remove oxyanions is of importance in environment protection. Structured LDHs are one new type of promising material due to their ability to capture organic and inorganic anions which can be used in water treatment. Calcined LDH powder is also an important type of material used in water treatment, since LDH can afford mixed metal oxides and has a special property called memory effect. The main advantages of LDHs over the conventional anionic exchange resins include their higher anion exchange capacity for certain oxyanions and their good thermal stability. Furthermore, LDHs can be fully regenerated in a short time for reuse. Lv et al. used MgAl-NO ${ }_{3}-\mathrm{LDH}$ powder [61] and calcined powder [62] to remove $\mathrm{F}^{-}, \mathrm{Cl}^{-}, \mathrm{Br}^{-}$, and $\mathrm{I}^{-}$, and concluded that the rate constant for exchange of nitrate anions by halide decreases in the order of $\mathrm{F}^{-}>\mathrm{Cl}^{-}>\mathrm{Br}^{-}>\mathrm{I}^{-}$, following pseudo-second order kinetic models. As described above in Section 2.4, the edge-on LDH films prepared on man-made supports exhibited the enhanced performances of regeneration for water treatment and membrane separation in comparison with the corresponding powdery LDH [58]. The novel and well-dispersed nanocrystals of LDH on the paper substrate without aggregation was considered to provide large specific surface area as well as good accessibility, underlying the superior adsorption performances of the film sample [58].

\subsection{Additives in Concrete and Flame Retardants}

Powdered LDH are one types of additives in concrete. Basically, CaAl-LDH has a general formula very similar to the AFm phase occurring in hydrated cement, being composed of positively charged layers $\left[\mathrm{Ca}_{2} \mathrm{Al}(\mathrm{OH})_{6}\right]^{+}$, and negatively charged interlayers $\left[\mathrm{X}^{-} \cdot n \mathrm{H}_{2} \mathrm{O}\right]^{-}$. AFm phase is generally believed to act as a crystal seed accelerating cement hydration, CaAl-LDHs have been, therefore, proposed as potential concrete hardening accelerators. Raki et al. synthesized CaAl-LDHs intercalated with nitrobenzoic acid, naphthalene-2, 6-disulfonic acid, and naphthalene-2 sulfonic acid, which were promising for future applications in cement and concrete science [63]. We recently reported that the specimens containing pure CaAl-LDHs, free of contamination by $\mathrm{CaCO}_{3}$, exhibited a greatly enhanced performance in respect to early compressive strength and early flexural strength; and the values increased by $61 \%$ and $71 \%$, respectively, compared to the pristine concrete specimen [34].

Powdery LDH is also commercially promising as an additive in flame retardants. Many flame retardants are considered harmful, having been linked to liver, thyroid, reproductive/developmental, and neurological effects. Currently, halogen-free alternatives are one active research area. LDHs and cationic clays (e.g., montmorillonite) have been widely investigated as additives in this context. 
Compared to other flame retardants, $\mathrm{LDH}$ is a new type of material due to high smoke suppression, nontoxicity or low toxicity. LDH is currently explored as a second generation flame retardant with enhanced properties by either modification of the layers or intercalation of different anions. Lin et al. found that a borate-pillared MgAl-LDH with ethylene vinyl acetate (EVA) as the polymer component was a promising flame retardant [64]. By tuning the composition of the layers, the flame retardancy of LDHs was steered. For example, ternary $\mathrm{ZnMgAl}-\mathrm{LDH}$ showed better flame retarding properties than binary $\mathrm{MgAl}-\mathrm{CO}_{3}{ }^{2-}$-LDHs [65].

\subsection{LDHs in Biology and Medicine}

Powdered LDHs have demonstrated to be one type of important and green carrier or host for genes and drugs due to the excellent biocompatibility and nontoxicity or low toxicity. MgAl-LDH is used as an important component of drugs, or as nanocarriers for delivery of drug and genes into cells $[5,8]$. Wei et al. demonstrated that LDH was able to be used as an effective nanocarrier by greatly enhancing the thermal- and photo-stabilities for L-Dopa [66] and L-Tyrosine [67], which are both unstable agents in storage or transport. Comprehensive investigations have been performed in Choy's group on the toxicity of LDH nanoparticles in vitro and in vivo in practical biological applications [5]. Interestingly, Lu's group recently reported an efficient LDH-based delivery for siRNA to mammalian cells in vitro. A pronounced down-regulation of protein expression upon LDH mediated siRNA transfection of HEK293T cells was observed [68].

\section{Conclusions}

In this feature article we have reviewed the morphology, preparation, and application of LDH structures. Basically, micro-/nanosized LDH powder is prepared through hydrothermal synthesis, conventional coprecipitation or scalable SNAS. The edge-on oriented LDH spheres (perpendicular to the tangent plane at the spherical surface) and films are fabricated via in situ growth, which is actually performed on the basis of hydrothermal synthesis, whereas the preparation of the face-on oriented LDH spheres and films is achieved by physical deposition methods (mainly consisting of LbL assembly, sol-gel spin-coating and the solvent evaporation technique) and hydrogen-bonding-based in situ growth. 1-D structured LDHs can be synthesized via control of LDH nucleation and growth in confined microsystems. In the point of view of applications, LDH powders and films are widely explored as catalysts and adsorbed materials, upon calcination and rehydration, the resulting activated structures exhibit enhanced performances. Powdery LDHs are typically used as addictives in flame retardants and concrete, and as nanocarriers for drugs, gene molecules, biomedical products, and functional molecules. A few big challenges, however, still remain, involving dedication to the problem of well-dispersed LDH nanoplatelets as additives in flame retardants and also to realize non-toxic, biocompatible and biodegradable LDH carries into practical applications for drug and gene delivery. Some perspectives could focus on preparing novel LDH micro-/nanostructures and developing novel applications in many fields such as separation, catalyst, drug and gene delivery, and electrode modifier energy harvesting devices, and also on the relationship between the novel structures and the enhanced properties of LDH micro-/nanostructures, as well as further understanding the specific structures 
prepared from confined nucleation and growth. These aspects would advance our knowledge of control LDH nanostructures, resultant properties and applications.

\section{Acknowledgements}

We would like to thank all the co-workers cited in the references below for their invaluable contributions to the work described here. The authors would like to thank D.G. Evans for inspiring discussions and helpful comments. This work was financially supported by the National Natural Science Foundation of China, the 111 Project (B07004), the Program for New Century Excellent Talents in Universities, and the Beijing Nova Program (2007B021).

\section{References and Notes}

1. Cavani, F.; Trifiròa, F.; Vaccaria, A. Hydrotalcite-type anionic clays: Preparation, properties and applications. Catal. Today 1991, 11, 173-301.

2. Rives, V. Layered Double Hydroxides: Present and Future; Nova Science Publishers: New York, NY, USA, 2001.

3. Khan, A.I.; O'Hare, D. Intercalation chemistry of layered double hydroxides: Recent developments and applications. J. Mater. Chem. 2002, 12, 3191-3198.

4. Sideris, P.J.; Nielsen, U.G.; Gan, Z.; Grey, C.P. Mg/Al ordering in layered double hydroxides revealed by multinuclear NMR spectroscopy. Science 2008, 321, 113-117.

5. Choi, S.-J.; Oh, J.-M.; Choy, J.-H. Human-related application and nanotoxicology of inorganic particles: Complementary aspects. J. Mater. Chem. 2008, 18, 615-620.

6. Oh, J.-M.; Biswick, T.T.; Choy, J.-H. Layered nanomaterials for green materials. J. Mater. Chem. 2009, 19, 2553-2563.

7. Williams, G.R.; O'Hare, D. Towards understanding, control and application of layered double hydroxide chemistry. J. Mater. Chem. 2006, 16, 3065-3074.

8. Xu, Z.P.; Qing, H.Z.; Gao, Q.L.; Ai, B.Y. Inorganic nanoparticles as carriers for efficient cellular delivery. Chem. Eng. Sci. 2006, 61, 1027-1040.

9. Guo, X.; Zhang, F.; Evans, D.G.; Duan, X. Layered double hydroxide films: Synthesis, properties and applications. Chem. Commun. 2010, 46, 5197-5210.

10. Roeffaers, M.B.J.; Sels, B.F.; Uji-i, H.; De Schryver, F.C.; Jacobs, P.A.; De Vos Johan Hofkens, D.E. Spatially resolved observation of crystal-face-dependent catalysis by single turnover counting. Nature 2006, 439, 572-575.

11. Xu, Z.P.; Stevenson, G.S.; Lu, C.Q.; Lu, G.Q.; Bartlett, P.F.; Gray, P.P. Stable suspension of layered double hydroxide nanoparticles in aqueous solution. J. Am. Chem. Soc. 2006, 128, 36-37.

12. Gursky, J.A.; Blough, S.D.; Luna, C.; Gomez, C.; Luevano, A.N.; Gardner, E.A. Particle-particle interactions between layered double hydroxide nanoparticles. J. Am. Chem. Soc. 2006, 128, 8376-8377.

13. Gunawan, P.; Xu, R. Synthesis of unusual coral-like layered double hydroxide microspheres in a nonaqueous polar solvent/surfactant system. J. Mater. Chem. 2008, 18, 2112-2120.

14. Du, Y.; Hu, G.; O'Hare, D. Nucleation and growth of oriented layered double hydroxides on polymer resin beads. J. Mater. Chem. 2009, 19, 1160-1165. 
15. Gunawan, P.; Xu, R. Direct assembly of snisotropic Layered Double Hydroxide (LDH) nanocrystals on spherical template for fabrication of drug-LDH hollow nanospheres. Chem. Mater. 2009, 21, 781-783.

16. Li, B.; He, J. Multiple Effects of Dodecanesulfonate in the Crystal Growth Control and Morphosynthesis of Layered Double hHdroxides. J. Phys. Chem. C 2008, 112, 10909-10917.

17. Li, L.; Ma, R.; Iyi, N.; Ebina, Y.; Takada, K.; Sasaki, T. Hollow nanoshell of layered double hyrdoxide. Chem. Commun. 2006, 42, 3125-3127.

18. Li, L.; Feng, Y.; Li, Y.; Zhao, W.; Shi, J. $\mathrm{Fe}_{3} \mathrm{O}_{4}$ core/layered double hydroxide dhell nanocomposite: Versatile magnetic matrix for anionic functional materials. Angew. Chem. Int. Ed. 2009, 48, 5888-5892.

19. $\mathrm{Hu}, \mathrm{G} . ;$ O'Hare, D. Unique layered double hydroxide morphologies using reverse microemulsion synthesis. J. Am. Chem. Soc. 2005, 127, 17808-17813.

20. De Jesús Martínez-Ortiz, M.; Lima, E.; Lara, V.; Vivar, J.M. Structural and textural evolution during folding of layers of layered double hydroxides. Langmuir 2008, 24, 8904-8911.

21. Chen, H.; Zhang, F.; Fu, S.; Duan, X. In situ microstructure control of oriented layered double hydroxide monolayer films with curved hexagonal crystals as superhydrophobic materials. Adv. Mater. 2006, 18, 3089-3093.

22. Gao, Y.F.; Nagai, M.; Masuda, Y.; Sato, F.; Seo, W.S.; Koumoto, K. Surface precipitation of highly porous hydrotalcite-like film on al from a zinc aqueous solution. Langmuir 2006, 22, 3521-3527.

23. Cho, S.; Jung, S.-H.; Jang, J.-W.; Oh, E.; Lee, K.-H. Simultaneous synthesis of Al-doped ZnO nanoneedles and zinc aluminum hydroxides through use of a seed layer. Cryst. Growth Des. 2008, 8, 4553-3558.

24. Liu, J.; Li, Y.; Huang, X.; Li, G.; Li, Z. Layered double hydroxide nano- and microstructures grown directly on metal substrates and their calcined products for application as Li-ion battery electrodes. Adv. Funct. Mater. 2008, 18, 1448-1458.

25. Zhang, F.; Zhao, L.; Chen, H.; Xu, S.L.; Evans, D.G.; Duan, X. Corrosion resistance of superhydrophobic layered double hydroxide films on aluminum. Angew. Chem. Int. Ed. 2008, 47, 2466-2469.

26. Guo, X.; Xu, S.; Zhao, L.; Lu, W.; Zhang, F.; Evans, D.G.; Duan, X. One-step hydrothermal crystallization of a layered double hydroxide/alumina bilayer film on aluminum and its corrosion resistance properties. Langmuir 2009, 25, 9894-9897.

27. Guo, X.; Zhang, F.; Xu, S.; Evans, D.G.; Duan, X. Preparation of layered double hydroxide films with different orientations on the opposite sides of a glass substrate by in situ hydrothermal crystallization. Chem. Commun. 2009, 44, 6836-6838.

28. Burleigh, M.C.; Jayasundera, S.; Spector, M.S.; Thomas, C.W.; Markowitz, M.A.; Gaber, B.P. A new family of copolymers: Multifunctional periodic mesoporous organosilicas. Chem. Mater. 2004, 16, 3-5.

29. Hu, G.; Wang, N.; O'Hare, D.; Davis, J. One-step synthesis and AFM imaging of hydrophobic LDH monolayers. Chem. Commun. 2006, 42, 287-289.

30. Du, L.C.; Qu, B.J. Preparation of LLDPE/MgAl-LDH exfoliation nanocomposites with enhanced thermal properties by melt intercalation. Chin. J. Chem. 2006, 24, 1342-1345. 
31. Hu, G.; Wang, N.; O’Hare, D.; Davis, A. Synthesis of magnesium aluminium layered double hydroxides in reverse microemulsions. J. Mater. Chem. 2007, 17, 2257-2266.

32. Zhao, Y.; Li, F.; Zhang, R.; Evans, D.G.; Duan, X. Preparation of layered double-hydroxide nanomaterials with a uniform crystallite size using a new method involving separate nucleation and aging steps. Chem. Mater. 2002, 14, 4286-4291.

33. Gu, Z.; Xiang, X.; Fan, G.; Li, F. Facile synthesis and characterization of cobalt ferrite nanocrystals via a simple reduction-oxidation route. J. Phys. Chem. C 2008, 112, 18459-18466.

34. Xu, S.L.; Chen, Z.; Zhang, B.; Yu, J.; Zhang, F.Z.; Evans, D.G. Facile preparation of pure $\mathrm{CaAl}$-layered double hydroxides and their application as a hardening accelerator in concrete. Chem. Eng. J. 2009, 155, 881-885.

35. Liu, Z.; Ma, R.; Osada, M.; Iyi, N.; Ebina, Y.; Takada, K.; Sasaki, T. Synthesis, anion exchange, and delamination of Co-Al layered double hydroxide: Assembly of the exfoliated nanosheet/polyanion composite films and magneto-optical studies. J. Am. Chem. Soc. 2006, 128, 4872-4880.

36. Yan, D.P.; Lu, J.; Wei, M.; Han, J.B.; Ma, J.; Li, F.; Evans, D.G.; Duan, X. Poly(p-phenylene) anionic derivative/layered double hydroxides ultra thin films with blue luminescence by layer-by-layer assembly. Angew. Chem. Int. Ed. 2009, 48, 3073-3076.

37. Han, J.B.; Lu, J.; Wei, M.; Wang, Z.L.; Duan, X. Heterogeneous ultrathin films fabricated by alternate assembly of exfoliated layered double hydroxides and polyanion. Chem. Commun. 2008, 44, 5188-5190.

38. Chen, D.; Wang, X.; Liu, T.; Wang, X.; Li, J. Electrically conductive poly(vinyl alcohol) hybrid films containing graphene and layered double hydroxide fabricated via layer-by-layer self-assembly. Appl. Mater. Interf. 2010, 2, 2005-2011.

39. Géraud, E.; Prévot, V.; Ghanbaja, J.; Leroux, F. Macroscopically ordered hydrotalcite-type materials using self-assembled colloidal crystal template. Chem. Mater. 2006, 18, 238-240.

40. Géraud, E.; Rafqah, S.; Sarakha, M.; Forano, C.; Prevot, V.; Leroux, F. Three dimensionally ordered macroporous layered double hydroxides: preparation by templated impregnation/ coprecipitation and pattern stability upon calcination. Chem. Mater. 2008, 20, 1116-1125.

41. Tao, Q.; Zhang, Y.; Zhang, X.; Yuan, P.; He, H.P. Synthesis and characterization of layered double hydroxides with a high aspect ratio. J. Solid State Chem. 2006, 179, 708-715.

42. Zhao, Y.; Jiao, Q.Z.; Ding, X.J.; Zhang, L.Q.; Liu, Y.Y. Hydrothermal synthesis of nanorods and nanowires of Mg/Al layered double hydroxides. Chem. Res. Chin. U. 2007, 23, 622-624.

43. Wu, H.; Jiao, Q.; Zhao, Y.; Huang, S.; Li, X.; Liu, H.; Zhou, M. Synthesis of Zn/Co/Fe-layered double hydroxide nanowires with controllable morphology in a water-in-oil microemulsion. Mater. Character. 2010, 61, 227-232.

44. Iyi, N.; Ebina, Y.; Sasaki, T. Water-swellable MgAl-LDH (Layered Double Hydroxide) hybrids: Synthesis, characterization, and film preparation. Langmuir 2008, 24, 5591-5598.

45. Wang, L.Y.; Li, C.; Liu, M.; Evans, D.G.; Duan, X. Large continuous, transparent and oriented self-supporting films of layered double hydroxides with tunable chemical composition. Chem. Commun. 2007, 43, 123-125.

46. Itaya, K.; Chang, H.C.; Uchida, I. Anion-exchanged hydrotalcite-like-clay modified electrodes. Inorg. Chem. 1987, 26, 624-626. 
47. Lee, J.H.; Rhee, S.W.; Jung, D.Y. Solvothermal ion exchange of aliphatic dicarboxylates into the gallery space of layered double hydroxides immobilized on Si substrates. Chem. Mater. 2004, 16, 3774-3779.

48. Lee, J.H.; Rhee, S.W.; Jung, D.Y. Orientation-controlled assembly and solvothermal ion-exchange of layered double hydroxide nanocrystals. Chem. Commun. 2003, 39, 2740-2741.

49. Lee, J.H.; Rhee, S.W.; Jung, D.Y. Selective layer reaction of layer-by-layer assembled layered double-hydroxide nanocrystals. J. Am. Chem. Soc. 2007, 129, 3522-3523.

50. Lee, J.H.; Rhee, S.W.; Nam, H.J.; Jung, D.-Y. Surface Selective deposition of PMMA on layered double hydroxide nanocrystals immobilized on solid substrates. Adv. Mater. 2009, 21, 546-549.

51. Matthew, S.; Yarger, E.M.P.S.; Choi, K.-S. Electrochemical synthesis of Zn-Al layered double hydroxide (LDH) films. Inorg. Chem. 2008, 47, 5859-5865.

52. Scavetta, E.; Ballarin, B.; Gazzano, M.; Tonelli, D. Electrochemical behaviour of thin films of $\mathrm{Co} / \mathrm{Al}$ layered double hydroxide prepared by electrodeposition. Electrochim. Acta 2009, 54, 1027-1033.

53. Khenifi, A.; Derriche, Z.; Forano, C.; Prevot, V.; Mousty, C.; Scavetta, E.; Ballarin, B.; Guadagnini, L.; Tonelli, D. Glyphosate and glufosinate detection at electrogenerated NiAl-LDH thin films. Analy. Chim. Acta 2009, 654, 97-102.

54. Zhang, F.; Sun, M.; Xu, S.L.; Zhao, L.; Zhang, B. Fabrication of oriented layered double hydroxide films by spin coating and their use in corrosion protection. Chem. Eng. J. 2008, 141, 362-367.

55. He, J.X.; Yamashita, S.; Jones, W.; Yamagishi, A. Templating effects of stearate monolayer on formation of Mg Al-Hydrotalcite. Langmuir 2002, 18, 1580-1586.

56. Lei, X.; Yang, L.; Zhang, F.; Evans, D.G.; Duan, X. Synthesis of oriented layered double hydroxide thin films on sulfonated polystyrene substrates. Chem. Lett. 2005, 34, 1610-1611.

57. Lv, Z.; Zhang, F.; Lei, X.; Yang, L.; Evans, D.G.; Duan, X. Microstructure-controlled synthesis of oriented layered double hydroxide thin films: Effect of varying the preparation conditions and a kinetic and mechanistic study of film formation. Chem. Eng. Sci. 2007, 62, 6069-6075.

58. Zhao, Y.; He, S.; Wei, M.; Evans, D.G.; Duan, X. Hierarchical films of layered double hydroxides by using a sol-gel process and their high adaptability in water treatment. Chem. Commun. 2010, 46, 3031-3033.

59. Zhang, L.H.; Li, F.; Evans, D.G.; Duan, X. Cu/Mn/Fe-layered double hydroxides and their mixed metal oxides: Physicochemical and catalytic properties in wet hydrogen peroxide oxidation of phenol. Ind. Eng. Chem. Res. 2010, 49, 5959-5968.

60. Lv, Z.; Zhang, F.; Lei, X.D.; Yang, L.; Xu, S.L.; Duan, X. In situ growth of layered double hydroxide films on anodic aluminum oxide/aluminum and its catalytic feature in aldol condensation of acetone. Chem. Eng. Sci. 2008, 63, 4055-4062.

61. Lv, L.; He, J.; Wei, M.; Duan, X. Kinetic studies on fluoride removal by calcined layered double hydroxides. Ind. Eng. Chem. Res. 2006, 45, 8623-8628.

62. Lv, L.; Li, L. Adsorption behavior of calcined layered double hydroxides towards removal of iodide contaminants. J. Radioanal. Nuclear Chem. 2007, 273, 221-226.

63. Raki, L.; Beaudoin, J.J.; Mitchell, L. Layered double hydroxide-like materials: Nanocomposites for use in concrete. Cement Concr. Res. 2004, 34, 1717-1724. 
64. Shi, L.; Li, D.Q.; Wang, J.R.; Li, S.F.; Evans, D.G.; Duan, X. Synthesis, flame-retardant and smoke-suppressant properties of a borate-intercalated layered double hydroxide. Clays Clay Miner. 2005, 53, 294-300.

65. Ling, S.; Li, D.Q.; Li, S.; Wang, J.; D.G., E.; Duan, X. The structure, flame retarding and smoke suppressing properties of Zn-Mg-Al-CO32-layered double hydroxides. Chin. Sci. Bull. 2005, 50, 1101-1104.

66. Wei, M.; Pu, M.; Guo, J.; Han, J.; Li, F.; He, J.; Evans, D.G.; Duan, X. Intercalation of L-dopa into layered double hydroxides: Enhancement of both chemical and stereochemical stabilities of a drug through host-guest interactions. Chem. Mater. 2008, 20, 5169-5180.

67. Wei, M.; Yuan, Q.; Evans, D.G.; Wang, Z.; Duan, X. Layered solids as a 'Molecular Container' for pharmaceutical agents: L-tyrosine-intercalated layered double hydroxides. J. Mater. Chem. 2005, 15, 1197-1203.

68. Ladewig, K.; Niebert, M.; Xu, Z.P.; Gray, P.P.; Lu, G.Q.M. Efficient siRNA delivery to mammalian cells using layered double hydroxide nanoparticles. Biomaterials 2010, 31, 1821-1829.

(C) 2010 by the authors; licensee MDPI, Basel, Switzerland. This article is an open access article distributed under the terms and conditions of the Creative Commons Attribution license (http://creativecommons.org/licenses/by/3.0/). 